\title{
Spinal perineurial and meningeal cysts
}

\author{
I. M. TARLOV \\ From the Hospital for Joint Diseases and Medical Center, and the Doctors' Hospital, \\ New York City, N.Y., U.S.A.
}

SUMMARY Perineurial cysts may be responsible for clinical symptoms and a cure effected by their removal. They do not fill on initial myelography but may fill with Pantopaque some time, days or weeks, after Pantopaque has been instilled into the subarachnoid space. Perineurial cysts arise at the site of the posterior root ganglion. The cyst wall is composed of neural tissue. When initial myelography fails to reveal an adequate cause for the patient's symptoms and signs referable to the caudal nerve roots, then about a millilitre of Pantopaque should be left in the canal for delayed myelography which may later reveal a sacral perineurial cyst or, occasionally, a meningeal cyst. Meningeal diverticula occur proximal to the posterior root ganglia and usually fill on initial myelography. They are in free communication with the subarachnoid space and are rarely in my experience responsible for clinical symptoms. Meningeal diverticula and meningeal cysts appear to represent a continuum. Pantopaque left in the subarachnoid space may convert a meningeal diverticulum into an expanding symptomatic meningeal cyst, as in the case described. Many cases described as perineurial cysts represent abnormally long arachnoidal prolongations over nerve roots or meningeal diverticula. In general, neither of the latter is of pathological significance. Perineurial, like meningeal cysts and diverticula, may be asymptomatic. They should be operated upon only if they produce progressive or disabling symptoms or signs clearly attributable to them. When myelography must be done, and this should be done only as a preliminary to a probable necessary operation, then patient effort should be made to remove the Pantopaque.

Sacral perineurial cysts have often since my original description (Tarlov, 1938, 1948, 1953) been confused with meningeal cysts and diverticula and with unusually long arachnoidal prolongations over nerve roots. Perineurial cysts are capable of producing clinical symptoms (Tarlov, 1948), and do not fill on initial myelography. Meningeal diverticula and prolongations of the subarachnoid space over the nerve roots do fill upon initial myelography and are, in my experience, not responsible for clinical symptoms unless the neck of the diverticulum develops a valvular mechanism. Transitions between meningeal diverticula and symptomatic meningeal cysts occur and may fill upon initial myelography.

It is important clearly to differentiate perineurial cysts, meningeal cysts, and diverticula, and unusually long arachnoidal prolongations over nerve roots, in order to avoid unnecessary operations. This communication is an attempt to eliminate the confusion about these lesions.

${ }^{1}$ Address: Department of Neurology, College of Physicians and Surgeons, Columbia University.

\section{PERINEURIAL CYSTS}

Perineurial cysts may be differentiated from meningeal cysts by their (1) failure to fill with contrast medium upon initial myelography, (2) location along the nerve root, and (3) microscopic features.

CLINICAL PICTURE All symptomatic perineurial cysts that $I$ have operated upon have been on the sacral nerve roots. However, I have seen one perineurial cyst arising from a thoracic nerve root and compressing the spinal cord. This cyst was removed by a colleague and full recovery followed. I have operated upon seven cases, aged 28 to 70 years. The symptoms, usually progressive, varied in duration from eight months to 20 years. There was a record of antecedent trauma in four cases. Pain or paraesthesia was present in every case. The dominant syndrome was referable to the caudal nerve roots, either sciatica, sacral or buttocks pain, vaginal or penile paraesthesia, or sensory changes over the buttocks, perineal area, and lower extremity. Urinary incontinence occurred in one patient. Weakness of 
a foot was present in two patients, in one of whom the ankle jerk was absent. In another case the ankle jerk was absent. Tenderness on firm pressure over the sacrum was present in three patients.

RADIOLOGICAL ABNORMALITIES Perineurial cysts may cause bony erosion (Fig. 1) of the sacrum but this fact does not provide differentiation from meningeal cysts as the latter may also erode bone (see Fig. 4).

On myelography, sacral perineurial cysts do not fill on initial examination. In none of my seven patients harbouring perineurial cysts and in none of my 13 cases of perineurial cysts found in a series of 90 cadaver dissections could communication with the subarachnoid space be demonstrated.

Although filling of perineurial cysts does not occur on initial myelography, I have observed two cases in which subsequent radiological examination revealed that contrast medium had entered the cyst cavity. This phenomenon of delayed myelographic filling of a perineurial cyst was reported by Schreiber $\stackrel{\mathbb{D}}{c}$ and Haddad (1951). The intervals between the initial and the delayed myelograms in my three cases of $\stackrel{\text { ? }}{=}$ perineurial cysts which filled on myelography were three months and four years. Further observations of are necessary to determine the earliest time after $\stackrel{0}{T}$ initial myelography at which these cysts become radiographically visible. The case reported by Basauri, Hudson, and Bardales (1969), though not verified histologically, suggests that 24 hours may be sufficient time for Pantopaque to enter a peri- $\overline{0}$ neurial cyst. The presence of large immobile col- 흠 lections (Fig. 1) of radiopaque material under the $\frac{\bar{\rho}}{T}$ sacrum or elsewhere corresponding anatomically to $\mathbb{\Phi}$ dorsal root ganglia strongly suggests perineurial cysts. By contrast, meningeal cysts when occurring ${ }^{\text {o }}$ along nerve roots, an uncommon location, do not ordinarily occupy the position of the dorsal root $\overrightarrow{\vec{\omega}}$ ganglia but occur proximal to these ganglia.

Thus, when initial myelography reveals no adequate cause for the patient's symptoms and signs?

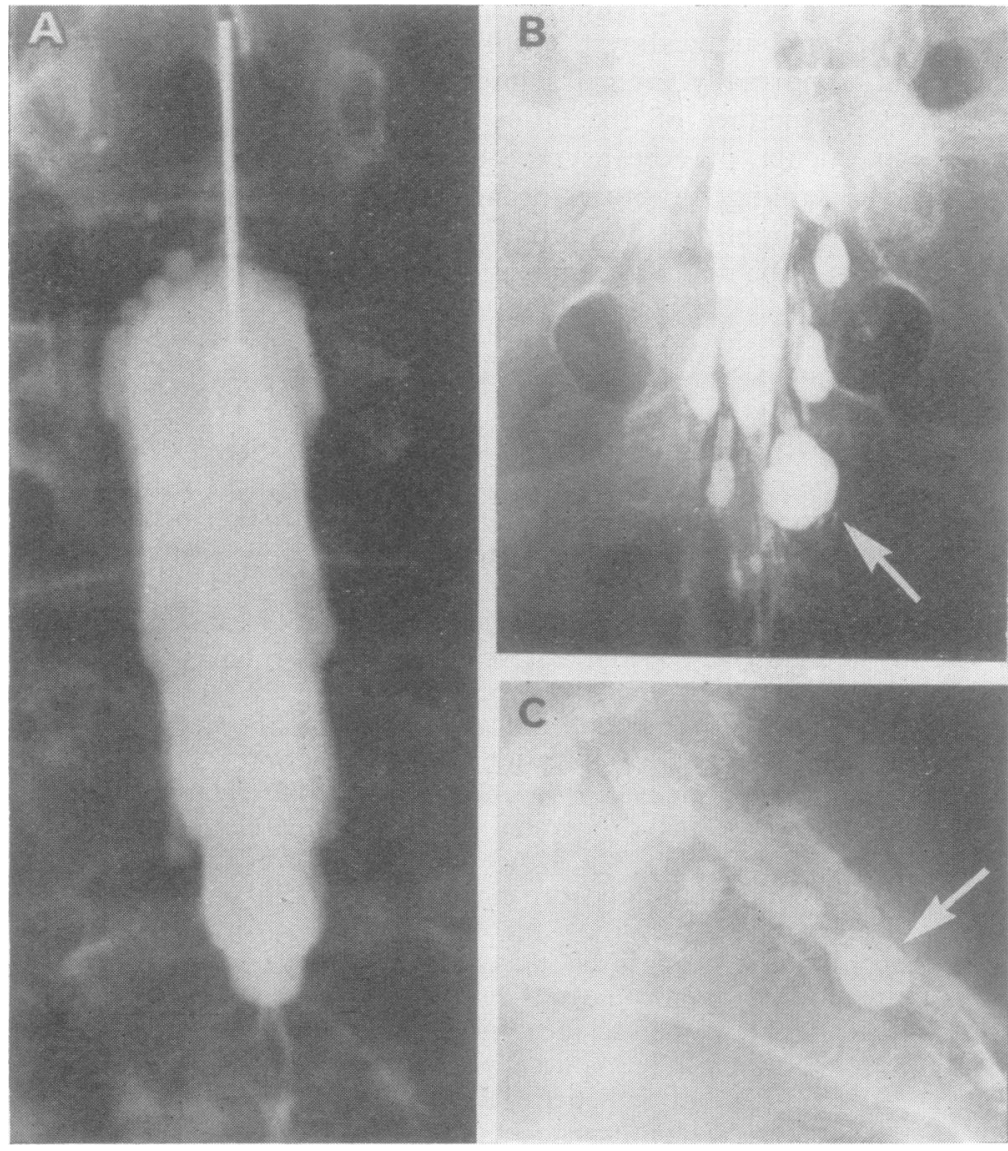

FIG. 1. Delayed myelographic filling of perineurial cyst. A. Normal initial myelogram, woman aged 46 complaining of sacrococcygeal and buttocks pain (nine months). B. Threemonth delayed A-P myelogram (Pantopaque was apparently unremovable) showing perineurial cyst (arrow). Other opacities were normal roots and ganglia at operation. C. Erosion of sacral body (arrow) by cyst. 
referable to the caudal nerve roots, about a millilitre of Pantopaque should be allowed to remain in the canal for delayed myelography which may later reveal a sacral perineurial cyst. The basis for delayed myelographic filling of perineurial cysts is that the perineurium is the peripheral extension of the arachnoid. In most individuals the communication between the subarachnoid and the subperineurial spaces is not free enough to allow immediate visualization of the perineurial spaces during myelography, though occasionally myelographic contrast medium does delineate nerve roots and even peripheral nerves (see Fig. 6). The pulsatile hydrostatic pressure of the cerebrospinal fluid appears to force Pantopaque into the perineurial space along nerve roots and peripheral nerves and occasionally into perineurial cysts, thus accounting for delayed myelographic visualization of these structures (Fig. 1). There was notching of the lower part of the thecal sac due to compression by the cyst in two cases.

Pathological features Perineurial and meningeal cysts may be clearly differentiated by their location and microscopically. Perineurial cysts occur along the nerve roots, at or distal to the junction of the posterior root and the dorsal ganglion (see Fig. 7). They begin in the perineurial space between the endoneurium derived from the pia mater and the perineurium formed by the arachnoid mater. Unlike meningeal cysts, at least a part of the lining of perineurial cysts contains nerve fibres (Fig. 2) and, at times, ganglion cells (Fig. 3). The entire cyst may be surrounded by nerve tissue. These cysts are usually seen on the sacral roots but they may occur at other levels (Tarlov, 1953). They may attain a diameter of $3 \mathrm{~cm}$, severely compressing or invading nerve roots, or they may be small without pathological significance. They are often multiple. Their pathogenesis in two of my cases studied at necropsy was clearly haemorrhage in a dorsal root ganglion (Tarlov, 1953). Other evidence points to a degenerative origin of some cysts. It seems likely that unusually long arachnoidal prolongations over nerve roots could form perineurial cysts if they were sealed off from the subarachnoid space by the inflammatory action of Pantopaque. Although abundant arachnoidal and perineurial tissue surrounds the nerve roots in their passage through the dura mater, proliferative arachnoidal changes have not been shown to cause these cysts (Tarlov, 1953). It is difficult to ascertain the number of recorded clinical cases of perineurial cysts because some of the cases so recorded appear to be either meningeal diverticula or cysts, or very long arachnoidal sleeves over nerve roots. Basauri et al. (1969) have, for example, recently described bilateral 'sacral cysts' which were clearly visualized 24 hours after an initially normal myelogram. These authors, as well as others (Strully, 1956), erroneously use the term 'diverticula of nerve root sheaths' synonymously with perineurial and arachnoidal cysts. Although histological verification is not provided (Basauri et al., 1969), the lesions described are probably perineurial cysts because they occurred distal to the point where the arachnoid becomes perineurium.

SURGICAL TREATMENT Perineurial cysts may be asymptomatic. When symptomatic, single perineurial cysts should be completely excised together with the posterior root and ganglion from which they arise. Complete excision of two dorsal and ventral nerve roots and ganglia (S2 and S3), one of which harboured a sacral perineurial cyst, was free from sequelae in the one patient in whom this was done. When dealing with three or more perineurial cysts arising from major nerve roots, removal of the domes consisting of perineurium and epineurium would probably be preferable to complete excision. Meningeal cysts can, of course, be completely excised without sacrificing any nerve fibres.

The seven patients upon whom I have operated for sacral perineurial cysts have obtained either complete or considerable relief from their symptoms, which were pain in five patients, paraesthesias and genitourinary disturbance in two patients. The surgical treatment was total excision of one or two cysts (S2 and S3, three patients), total excision of one cyst and excision of the dome of the other (S2, bilaterally, one patient), excision of the domes of the cyst (S2 and S3, unilaterally in one patient and bilaterally in the other), excision of the dome of one cyst and incision and drainage of the other (S2, bilaterally, one patient). The follow-up period varied from 18 months to 19 years. Total excision gave the best results.

\section{MENINGEAL CYSTS}

Meningeal cysts initially develop as outpouchings from the subarachnoid space. They may be either extradural, in which case they have a fibrous wall lined with arachnoid mater, or intradural and hence leptomeningeal. Meningeal cysts cannot be differentiated on plain x-rays from perineurial cysts: both may erode bone (Figs. 1 and 4 ). The extradural variety may be spontaneous or less commonly traumatic. Occasionally symptomatic meningeal cysts may arise from meningeal diverticula as illustrated by the following case.

D.M., aged 52, had for two years been troubled by increasing difficulty in initiating defaecation and urination. 

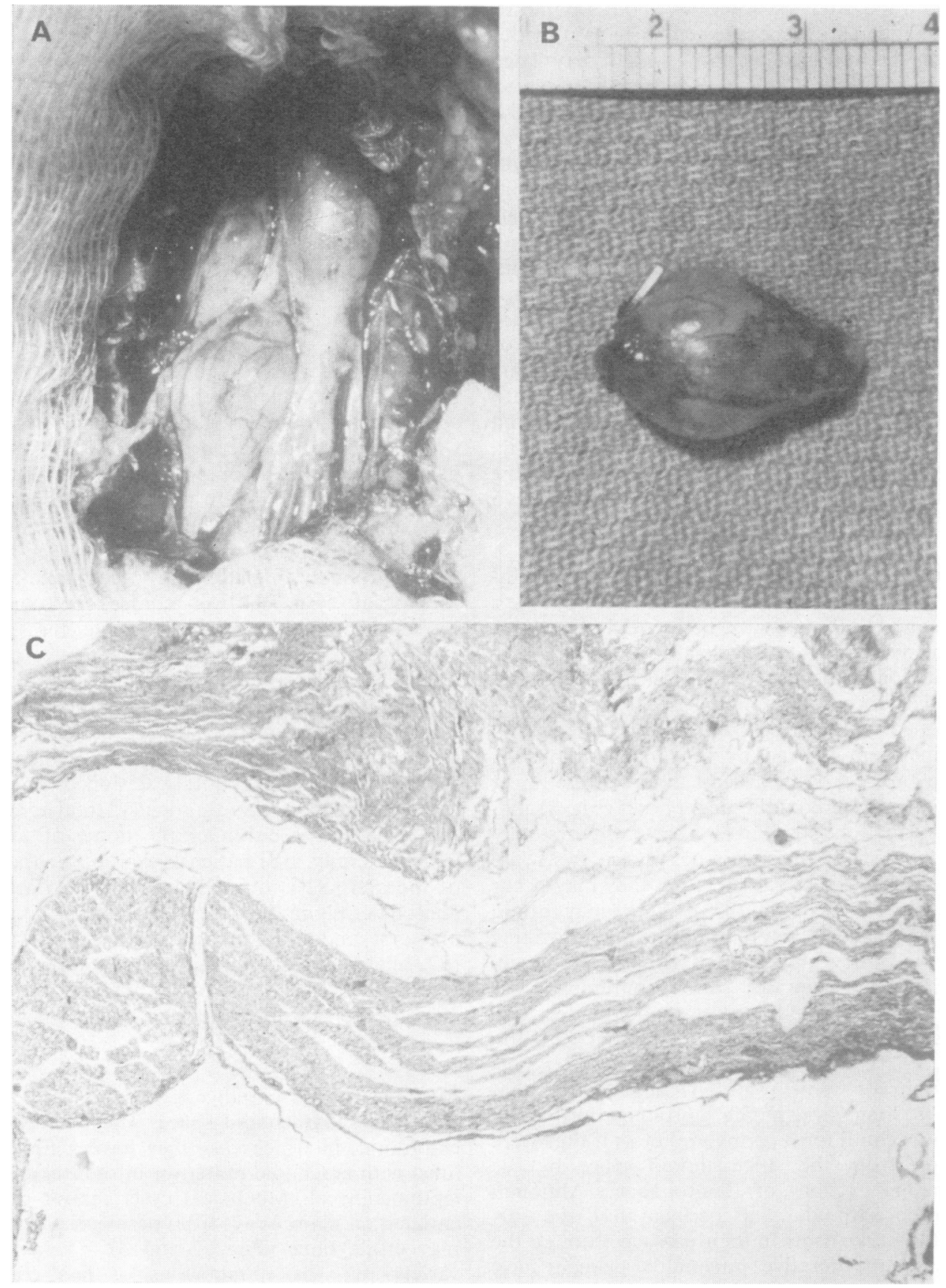

FIG. 2. A. S2 perineurial cyst, shown in A-P view in Fig. 1 at operation. B and C. Gross and histological specimens. A root-fascicle is in the centre of the cyst. Perineurial part of cysts is usually connected with intraneural, as in Fig. 3. $H$ and $E, \times 16$. 

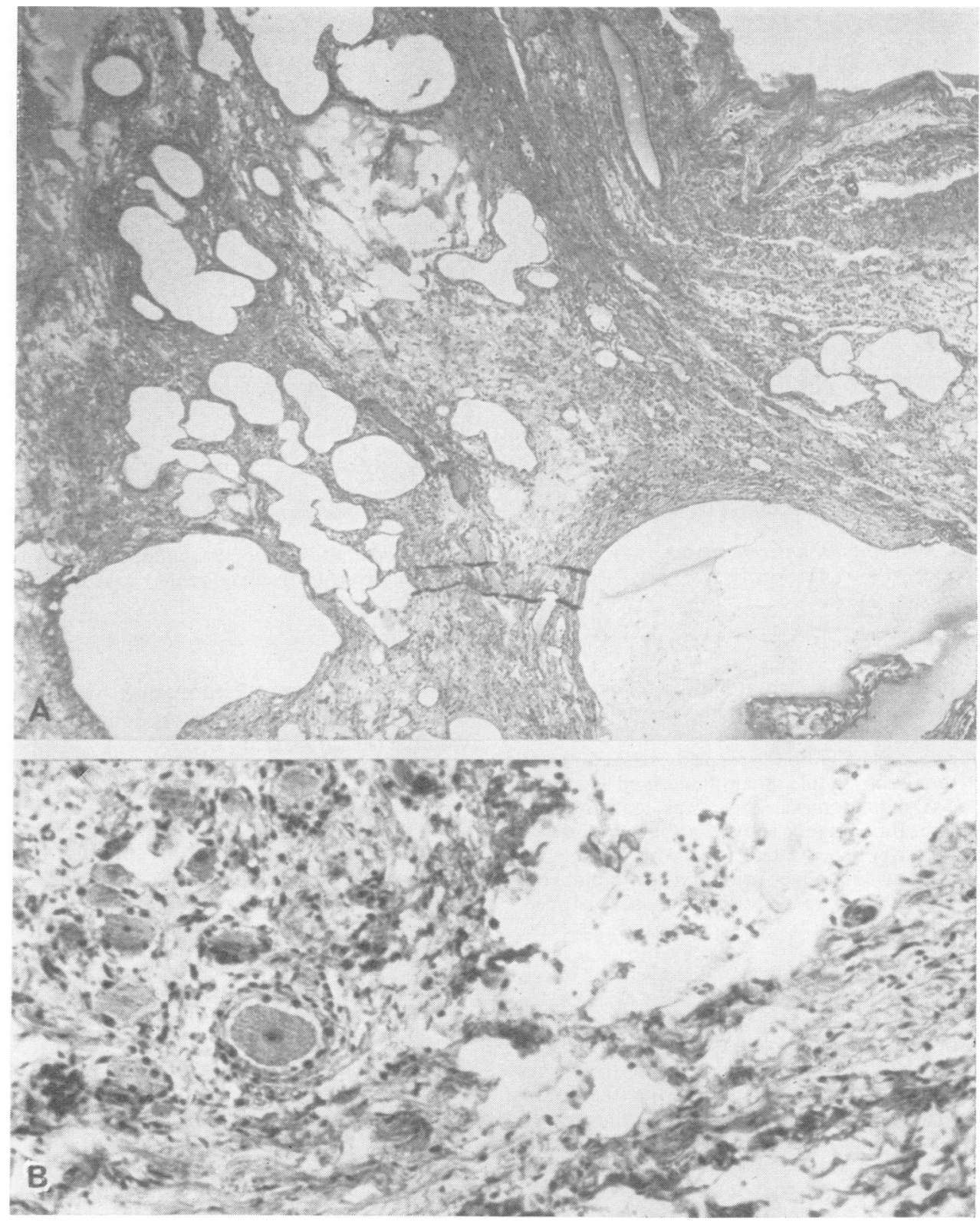

FIG. 3. Intraneural part of cyst shown in Fig. 2. A. multiloculation. B. Degeneration of tissue with cyst formation in region of posterior root ganglion. These cysts are frequently multiloculated. Therefore complete removal of the cyst along with the posterior root from which it arises is preferable simply to unroofing the cyst, though the latter procedure may be necessary in cases with multiple cysts. $H$ and $E$ : A, $\times 40 ; B, \times 120$. (Case referred by Drs. M. Lazansky and B. Golub.)

The straining required to initiate these acts often took over an hour and frequently produced transitory numbness of either lower limb. Five years previously a herniated intervertebral disc had been removed from L4-L5 on the left side because of low back pain and sciatica. Lumbosacral radiographs were then said to 

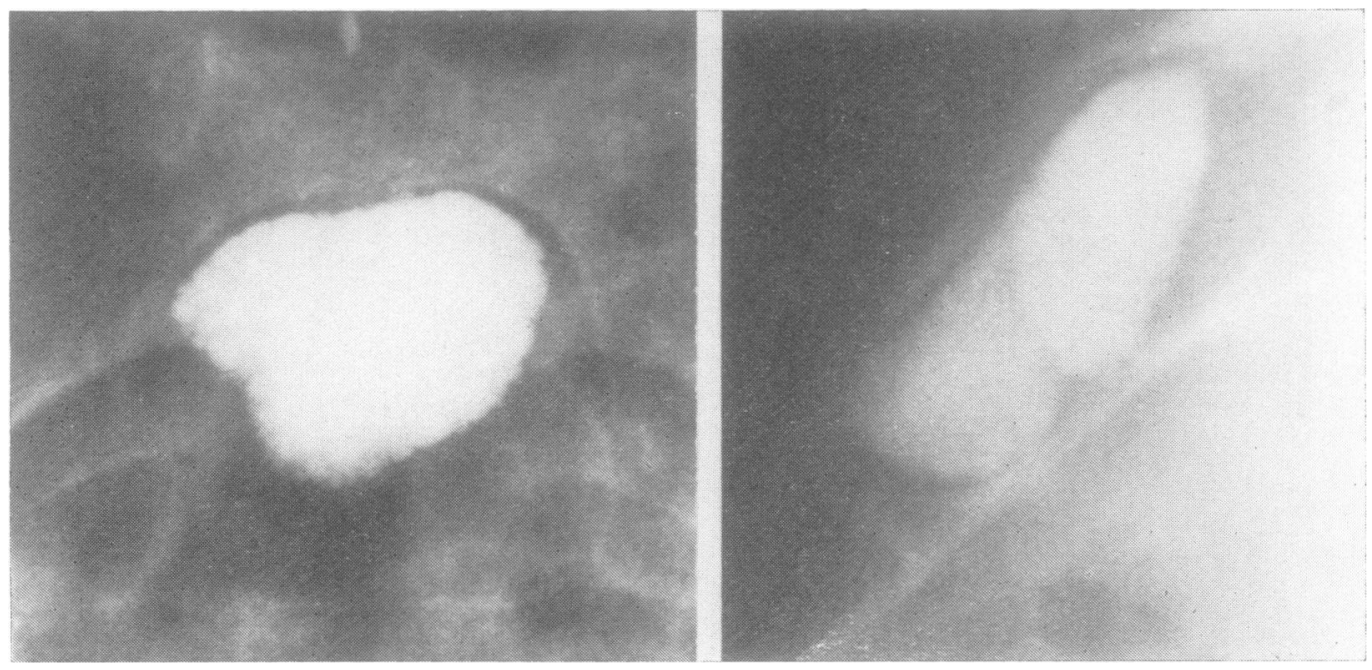

FIG. 4. Radiographs, anteroposterior and lateral views, showing sacral meningeal cyst outlined by Pantopaque introduced for myelography five years previously. Posterior sacral arch and body are thinned by cyst. Case D.M. (see text).

have been normal. A myelogram had been done at that time, after which the ethyl iophenyludecylate (Pantopaque) had not been removed.

Neurological examination revealed relaxed rectal sphincter tone and saddle anaesthesia and analgesia (S3, 4, and 5 dermatomes). There was tenderness on sacral pressure. Radiographs showed a thinned posterior sacral arch and a widened sacral canal which harboured a large, rounded immobile collection of radiopaque material (Fig. 4). The preoperative diagnosis was meningeal rather than perineurial cyst because of its central location.

Sacral laminectomy was done under general anaesthesia. The posterior sacral arch was paper-thin. Underneath there was a thin-walled translucent cyst filling the enlarged canal. Compressing the cyst did not collapse it, nor did compressing the thecal sac increase the size or tension of the cyst. No fluid escaped when the fibrous pedicle was transected.When the cyst wall was ruptured, a large amount of yellowish-white oily fluid escaped. The cyst could easily be separated from the cauda equina nerve roots and was completely removed.

Histologically, the cyst wall was composed of inflamed, thickened arachnoid and dura with granulation tissue (Fig. 5).

The patient made a good recovery. Her preoperative sacral sensory disturbance had vanished a month after operation and her urinary difficulty cleared two months later. Slight difficulty in initiating defaecation had persisted when she was examined 10 months postoperatively.

COMMENT This case illustrates an instance where a meningeal diverticulum developed into a symptomatic meningeal cyst apparently as a result of the inflammatory effect of Pantopaque. Although Panto paque appears to be the most innocuous mediurō $\rightarrow$ available for satisfactory myelography, its meningeaf irritative effects are well known (Peacher and Robertson, 1945; Tarlov, 1945). Mason and Raå (1962) have reported the death of a young man from undoubted diffuse aseptic meningitis, proved necropsy after myelography.

The cyst could not have resulted from trauma at the time of her first operation because the lower end of the thecal sac at the site of the cyst's origin had not then been exposed. The diverticulum may well have remained asymptomatic had not Pantopaque entered and sealed its communication with the subarachnoid space. Removal of the Pantopaque at the time of the myelogram or, if this were impossible, at operation might have prevented the formation of the cyst.

Occasionally a meningeal diverticulum arising from dural root sleeves may produce pressure effects even though there is some communication with the subarachnoid space. This seems to have been so in Schurr's (1955) patient in whom the 'valvular nature of the aperture had evidently prevented the cerebrospinal fluid from returning to the subarachnoid space...'

EXTRADURAL MENINGEAL CYSTS, SPONTANEOUS

Gortvai (1963) collected 46 cases of extradural meningeal cysts from the literature and added five of 

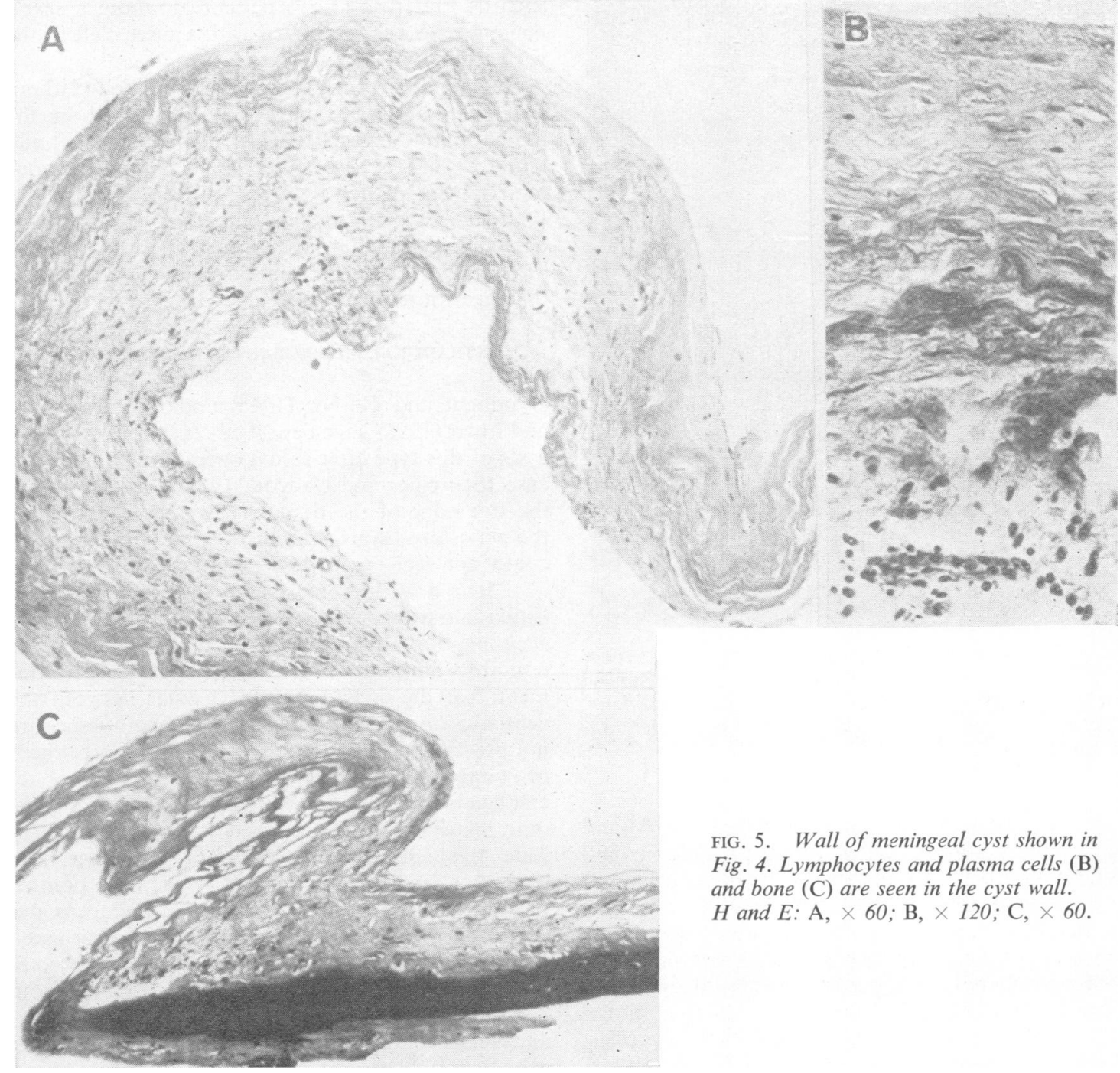

his own. Eroded pedicles, widened interpedicular spaces, or scalloped vertebral bodies were frequently seen in the radiographs. In Gortvai's patients the cysts, probably structurally transitional to diverticula, filled with contrast medium making a correct preoperative diagnosis possible. In my opinion, when immediate myelographic filling takes place the lesions are usually of no pathological significance and I would consider these as diverticula. In such cases it is important to try to remove the Pantopaque lest the diverticula be converted into expanding cysts as in my patient herein reported.

Dastur (1963) described three cases in which anteroposterior radiographs revealed rounded para-
FIG. 5. Wall of meningeal cyst shown in Fig. 4. Lymphocytes and plasma cells (B) and bone $(\mathrm{C})$ are seen in the cyst wall. $H$ and $E: \mathrm{A}, \times 60 ; \mathrm{B}, \times 120 ; \mathrm{C}, \times 60$. vertebral shadows caused by the lateral extension of these cysts, another diagnostic aid. This does not occur with perineurial cysts. Meningeal cysts occur most commonly in adolescence but they may appear in all decades through the sixth throughout the vertebral column, most commonly at thoracic levels. They arise from the midline or along the dural sleeve over the nerve root, one cyst arising from the anteromedial aspect of the dura. They communicated with the subarachnoid space in 12 of the collected cases. The fluctuation of symptoms that frequently occurs in these patients is thought to be due to the periodic self-emptying of the cysts.

The occurrence of extradural meningeal cysts in 


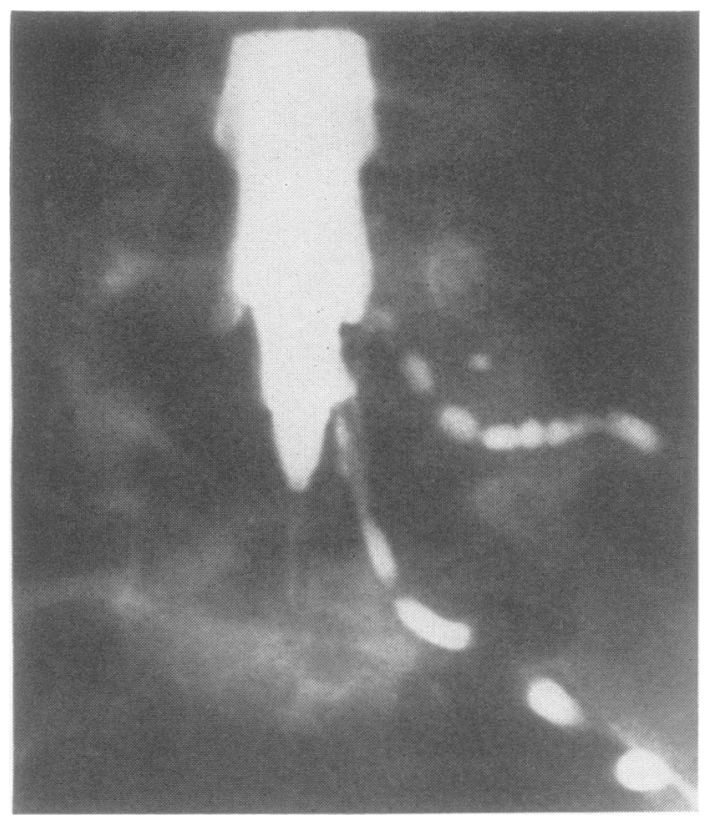

FIG. 6. Pantopaque along prolonged subarachnoid space over L5-S1 roots and peripheral nerves, anatomical variation without significance. Tilting patient head-down, Pantopaque returned to subarachnoid space and was removed.

three members of one family (Bergland, 1968) and in a 20-month-old baby (Nugent, Odom, and Woodhall, 1959) favours their congenital origin, at least in some cases. Possibly they result from failure of fusion of mesenchymal structures surrounding the neural tube, an embryological defect similar to that underlying spina bifida. Extradural cysts and extradural meningeal diverticula occurring in the sacral region have been called sacral meningoceles. To refer to meningoceles or diverticula as cysts is inaccurate because, unless the necks of these lesions become occluded, they are not by definition cysts. However, transitions between these types of lesions occur in which there is minimal communication between the subarachnoid space and the cyst or diverticulum. To call meningoceles meningeal diverticula is, strictly speaking, accurate, although by common usage meningocele refers to a visible sac of meninges protruding through a defect in the skull or spinal column. However, Wilson and Wakely (1932) have described a patient whose back appeared normal, yet in whom there was a large lumbosacral meningocele protruding beyond the laminar openings burrowing under the erector spinae muscles. Also there are transitional cases such as Enderle's (1932) patient in whom a sacral meningocele was associated with a mere cleft in the vertebral arch.

Ganglion cysts such as are commonly found about the wrist and hand have been described in the lumbar extradural space (Kao, Uihlein, Bickel, and Soule, 1968). These cysts, filled with gelatinous material surrounded by a wall of fibrous tissue, are thought to result from degeneration of connective tissue adjacent to a synovial joint. They are rare and $\vec{\Rightarrow}$ are not connected with, though they may compress, meninges or nerve roots.

\section{EXTRADURAL MENINGEAL CYSTS, TRAUMATIC}

Hyndman and Gerber (1946) and recently Miller \& and Elder (1968) have described cervical and lumbar $\vec{\circ}$ cysts of this type after spinal operation. In one such case (Schreiber and Haddad, 1951) a 'silver clip on $\bar{\omega}$ the free edge of the dura ... . was responsible for the persisting fistula inasmuch as the dural margins could not seal completely about the foreign body ... It is a well-known fact that with a persistent fistula admitting spinal fluid into the soft tissue, the accumulation of fluid soon becomes encased by $\vec{\xi} \omega$ non-absorbing wall. . . It is because of this develope ment that the construction of fistulas between the ventricles or subarachnoid spaces and soft tissues afe not practical or of permanent value in the treatment $\mathbb{D}$ of congenital hydrocephalus.' These cases re $\mathbb{\Phi}$ emphasize the importance of avoiding damage to the dura mater and to the necessity as far as possible of water-tight closure when the dura mater has been $\vec{\theta}$ opened. Schreiber and Haddad (1951) have pointed out that the inadvertent nicking of the dura mater while teasing it away from a neoplasm or a herniated intervertebral disc may set the stage for this complication in which fluid may enter an arachnoid herniation (true cyst) or a cavity (false cyst) (Fig. 7) and subsequently lose its communication with the subarachnoid space.

Meningeal diverticula may occur at the site of avulsed nerve roots (Fig. 8).

\section{INTRADURAL LEPTOMENINGEAL CYSTS}

Intradural leptomeningeal cysts are uncommon if one excludes from this category cases of cystic arachnoiditis. A striking example of these lesions $O$ was Hoffmann's (1960) 6 year old boy who recovered from quadriplegia after removal of an intradural 윽 arachnoidal cyst at C3. In a series of 41 cases of $>$ intraspinal tumours occurring below the age of 15 years, Svien, Thelen, and Keith (1954) found two N cases of intradural extramedullary arachnoidal cysts, the signs and symptoms being indistinguishable 


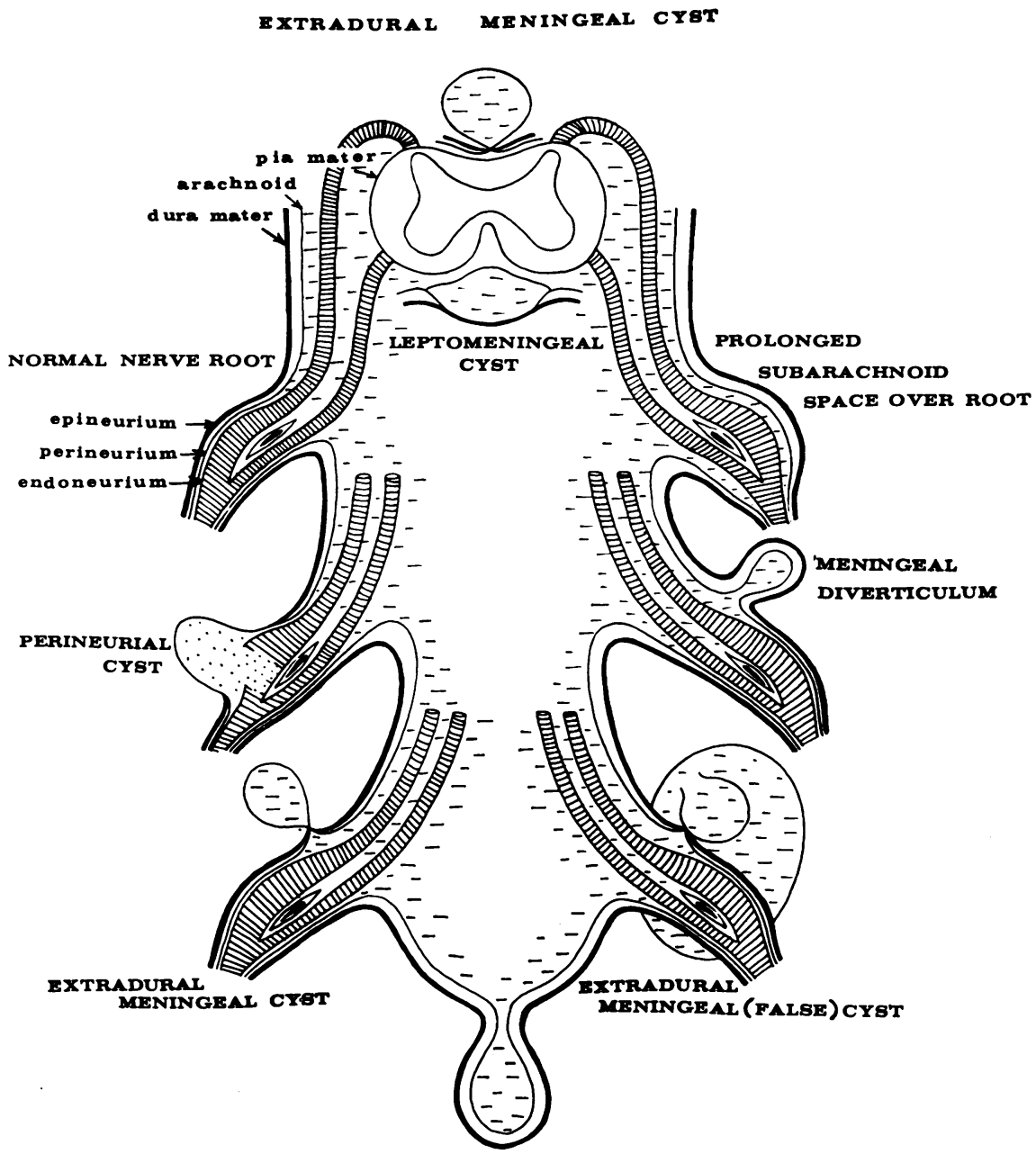

Manderal DrVarticulum F.T.

F IG. 7.

Diagram illustrating värious types of non-neoplastic, non-parasitic cysts, and diverticula of meninges and nerve roots. Top: extradural meningeal cyst, usually midline, covered by markedly thinned dura, or it may represent herniated arachnoid through dural defect. Leptomeningeal cyst, seen below cord figure, composed of arachnoid. Upper left: normal nerve root. Note that arachnoid continuation is perineurium and that subarachnoid space at root sleeve is continuous with potential space beneath perineurium, accounting for occasional myelographic filling of perineurial cysts. Middle left: perineurial cyst. Note that this lies at level of posterior root ganglion. Cyst wall contains neural elements. Space within cyst may have potential communication with subarachnoid space, as shown, allowing for delayed but not immediate myelographic filling. Lower left: extradural meningeal cyst. Note that this lies proximal to posterior root ganglion. Bottom: meningeal diverticulum. Sealing off neck of diverticulum produces cyst, as in case described in text. Lower right: extradural meningeal (false) cyst. May arise from rupture of extradural meningeal cyst, produced at operation by nicking the dura. Middle right: meningeal diverticulum. Lies proximal to posterior root ganglion. Ordinarily of no pathological significance, but if communication with subarachnoid space becomes sealed off may form symptomatic extradural meningeal cyst. Upper right: prolongation of subarachnoid space over nerve root, a rather frequent finding of no pathological significance. 


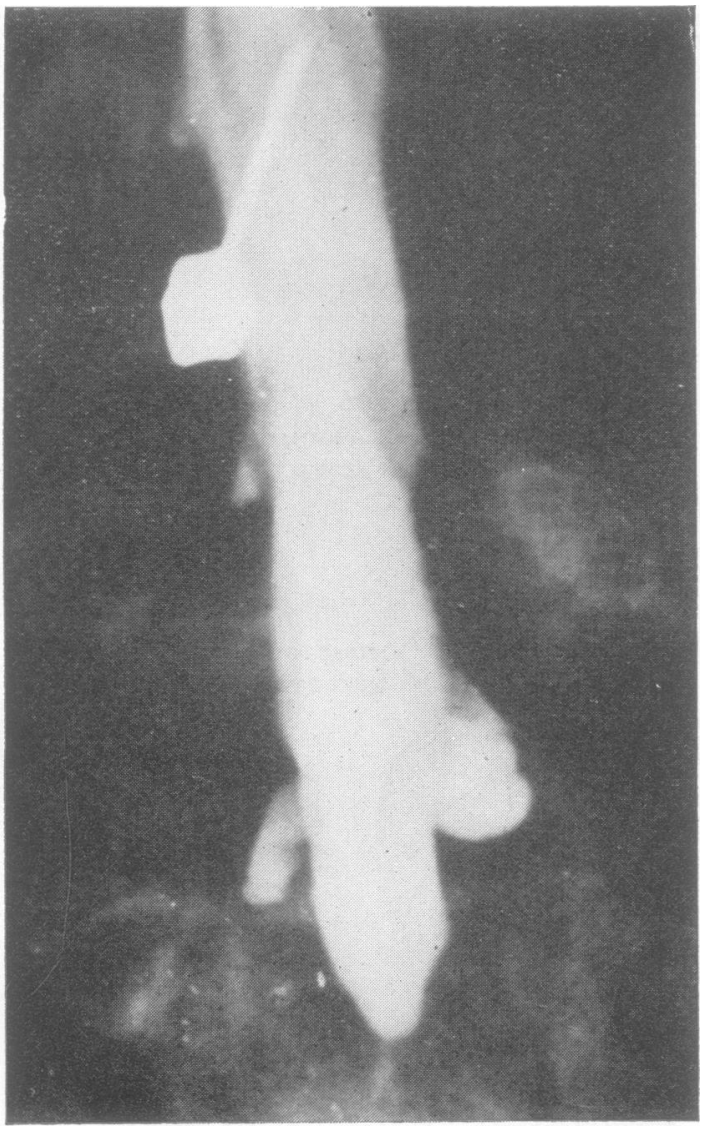

FIG. 8. Meningeal diverticulum in an 18-year-old woman who developed a right foot drop immediately after being hit by a truck. She sustained fractures of the pelvis, sacrum, body of L3, and the transverse processes of L4 and L5 vertebrae on the right side, the side of the myelographic abnormality at L5-S1. Although this patient was not operated upon, I have confirmed at operation similar meningeal diverticula (traumatic meningocele) in the cervical region at the site of avulsed nerve roots (Tarlov and Day, 1954). In such cases the accumulation of Pantopaque may extend lateral to the usual confines of the subarachnoid space. (Case referred by Dr. O. Kestler.)

from those of other spinal cord tumours. Lombardi and Morello (1963) culled 16 confirmed cases of cervical, thoracic, or lumbar intradural arachnoidal cysts from the literature and added their own case of a woman aged 59 years. Microscopic examination revealed normal arachnoid tissue. It appears likely that '. . . a preformed arachnoidal pocket, due to a developmental error, may lose free communication, bulge, and act as a tumor.' Teng and Rudner (1960) described fortuitous myelographic findings suggest- ing arachnoidal pockets which filled with Pantopaque in certain positions and emptied in other positions. They suggested that these formations might represent symptomless leptomeningeal 'cysts' that could develop a one-way valve mechanism and thereby produce cord compression. Such an interpretation should be considered with extreme caution. These presumed pockets might result from normal arachnoidal trabeculae, or adhesions that temporarily arrest the flow of Pantopaque. Every effort should be made in all meningeal diverticula or 'cysts' to dislodge the opaque medium from its 'pocket' by positioning the patient and by gentle back-thumping. Because of the viscosity of the Pantopaque, even its retention in the pocket would not preclude its normal communication with the subarachnoid space. Operation should not be undertaken in any such $\vec{\circ}$ case, unless the patient's symptoms are significantly handicapping and clearly attributable to the myelographic defect (Fig. 9).

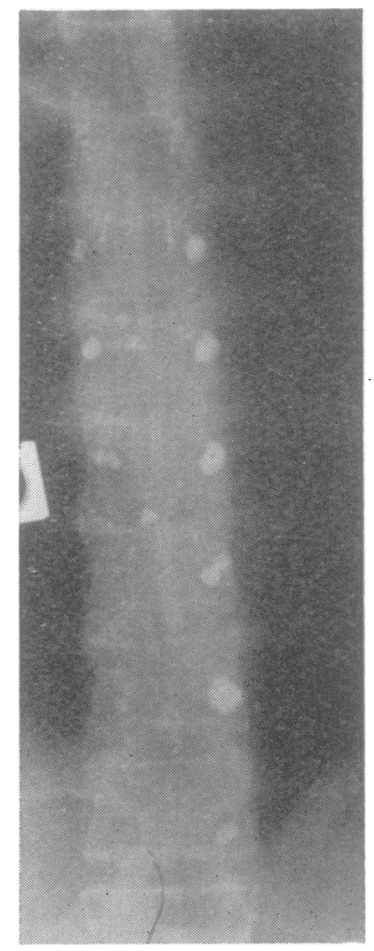

FIG. 9. Myelogram showing incidental finding of Pantopaque collections probably within distended prolongations of 윽 the subarachnoid space over the nerve roots. Their multiplicity and high degree of symmetry favour this interpretation rather than that of meningeal diverticula. They are of no pathological significance. (Case of Dr. Raymond N. O Kjellberg, Massachusetts General Hospital, Boston, Massachusetts).

(1)




\section{ADDENDUM}

Holt and Yates (1964, Cervical nerve root 'cysts', Brain, 87, 481-490) have described small cystic lesions of the posterior root at its junction with the dorsal ganglion in 36 of 120 cervical spines from elderly patients. From their description the lesions were clearly perineurial cysts. It is noteworthy that two of their patients were known to have complained of pain and paraesthesia of the upper limb. 'It seems likely that further experience will reveal their presence on other spinal as well as on cranial sensory roots and ganglia' (1953, Tarlov, Sacral Nerve-Root Cysts. Thomas: Springfield, Illinois).

\section{REFERENCES}

Basauri, L., Hudson, H., and Bardales, A. (1969). Diverticuli of the nerve root sheaths. J. Neurosurg., 31, 580-582.

Bergland, R. M. (1968). Congenital intraspinal extradural cyst. J. Neurosurg., 28, 495-499.

Dastur, H. M. (1963). The radiological appearances of spinal extradural arachnoid cysts. J. Neurol. Neurosurg. Psychiat., 26, 231-235.

Enderle, C. (1932). Meningocele intrasacrale occulto (rivelato con la mielografia). Riv. Neurol., 5, 418-423.

Gortvai, P. (1963). Extradural cysts of the spinal canal. J. Neurol. Neurosurg. Psychiat., 26, 223-230.

Hoffmann, G. T. (1960). Cervical arachnoidal cyst. $J$. Neurosurg., 17, 327-330.

Hyndman, D. R., and Gerber, W. F. (1946). Spinal extradural cysts, congenital and acquired, report of cases. J. Neurosurg., 3, 474-486.

Kao, C., Uihlein, A., Bickel, W., and Soule, E. (1968). Lumbar intraspinal extradural ganglion cyst. J. Neuro- surg., 29, 168-172.

Lombardi, G., and Morello, G. (1963). Congenital cysts of spinal membranes and roots. Brit. J. Radiol., 36, 197-205.

Mason, M. S., and Raaf, J. (1962). Complications of Pantopaque myelography. J. Neurosurg., 19, 302-311.

Miller, P. R., and Elder, F. W., jnr. (1968). Meningeal pseudocysts (meningocele spurius) following laminectomy. J. Bone Jt. Surg., 50A, 268-276.

Nugent, G. R., Odom, G. L., and Woodhall, B. (1959). Spinal extradural cysts. Neurology (Minneap.), 9, 397-406.

Peacher, W. G., and Robertson, R. C. L. (1945). Pantopaque myelography: Results, comparison of contrast media, and spinal fluid reaction. J. Neurosurg., 2, 220-231.

Schreiber, F., and Haddad, B. (1951). Lumbar and sacral cysts causing pain. J. Neurosurg., 8, 504-509.

Schur, P. H. (1955). Sacral extradural cyst: an uncommon cause of low back pain. J. Bone Jt. Surg., 37B, 601-605.

Strully, K. J. (1956). Meningeal diverticula of sacral nerve roots (perineurial cysts). J. Amer. med. Ass., 161, 1147 1152.

Svien, H. J., Thelen, E. P., and Keith, H. M. (1954). Intraspinal tumors in children. J. Amer. med. Ass., 155, 959-961.

Tarlov, I. M. (1938). Perineurial cysts of the spinal nerve roots. Arch. Neurol. Psychiat. (Chic.), 40, 1067-1074.

Tarlov, I. M. (1945). Pantopaque meningitis disclosed at operation. J. Amer. med. Ass., 129, 1014-1017.

Tarlov, I. M. (1948). Cysts (perineurial) of the sacral roots. J. Amer. med. Ass., 138, 740-744.

Tarlov, I. M. (1953). Sacral Nerve-Root Cysts: Another Cause of the Sciatic or Cauda Equina Syndrome. Charles C. Thomas: Springfield, Ill.

Tarlov, I. M., and Day, R. (1954). Myelography to help localize traction lesions of the brachial plexus. Amer. J. Surg., 88, 266-271.

Teng, P., and Rudner, N. (1960). Multiple arachnoid diverticula. Arch. Neurol. (Chic.), 2, 348-356.

Wilson, S. A. K., and Wakeley, C. P. G. (1932). Occult lumbosacral meningocele. J. Neurol. Psychopath., 13, 45-49. 